\title{
Dimensionamento do pessoal de enfermagem das unidades de internação do Hospital São Paulo*
}

\author{
Mari Sahamura Matsushita ${ }^{1}$ \\ Nilce Piva Adami \\ Maria Isabel Sampaio Carmagnani ${ }^{3}$
}

\begin{abstract}
Matsushita MS, Adami NP, Carmagnani MIS. Dimensionamento do pessoal de enfermagem das unidades de internação do Hospital São Paulo. Acta Paul Enferm 2005; 18(1):9-19.

RESUMO: Este estudo descritivo foi realizado no Hospital São Paulo, visando dimensionar o pessoal de enfermagem das unidades de internação desta instituição, e comparar os resultados encontrados com o quadro de pessoal de enfermagem existente. A coleta de dados foi realizada no ano de 2002, nas 40 unidades de internação, onde foram estudadas a classificação de pacientes e as ausências não previstas dos profissionais de enfermagem do ano anterior, sendo aplicado o método de dimensionamento de pessoal proposto por Gaidzinski'. As principais conclusões foram: os pacientes internados demandaram cuidados mínimos $(30,8 \%)$, seguidos pelos cuidados intermediários $(27,5 \%)$, cuidados de alta dependência $(22,0 \%)$, cuidados intensivos (12\%), e cuidados semi-intensivos (7,6\%). O tempo de assistência de enfermagem destinado aos pacientes classificados foi maior para os de cuidados intensivos (30,9\%).Cerca de $50,0 \%$ das ausências não previstas das enfermeiras foram referentes às licenças maternidade e as dos técnicos/auxiliares de enfermagem foram distribuídas de forma eqüitativa entre faltas, licenças médicas e licenças maternidade. A comparação entre os quadros de pessoal de enfermagem existente e o calculado demonstrou carência de 76 enfermeiras e excesso de 97 técnicos/auxiliares de enfermagem.
\end{abstract}

Descritores: Recursos humanos de enfermagem; Recursos humanos de enfermagem no hospital; Reorganização de recursos humanos; Administração de recursos humanos em hospitais; Dimensionamento de pessoal

- Artigo recebido em 12/08/04 e aprovado em 28/09/04

\section{INTRODUÇÃO}

A função dos hospitais foi se modificando ao longo do tempo, acompanhando a evolução da medicina. Atualmente, o hospital é considerado a mais complexa das organizações de saúde, face à diversidade de profissionais, usuários, tecnologias, relações interpessoais, processos de trabalho, formas de organização e espaços que alberga ${ }^{(2)}$
O serviço de enfermagem, como parte integrante das organizações hospitalares, tem acompanhado a evolução ocorrida nesta área, desenvolvendo uma estrutura de trabalho dentro de modelos cientificamente fundamentados. No entanto, um dos pontos polêmicos, quando se analisa ou se estrutura um serviço de enfermagem, é o dimensionamento de recursos humanos para assistir à clientela $^{(3)}$.
Assim, o provimento dos recursos humanos de enfermagem de hospitais públicos e privados, merece um amplo estudo devido à sua complexidade, face às variáveis envolvidas no seu cálculo. Os trabalhos publicados nas últimas cinco décadas do século XX têm evidenciado uma evolução nos modelos propostos para o dimensionamento de pessoal contemplando, atualmente, maior número de variáveis, permitindo melhor

\footnotetext{
* Trabalho extraído da Dissertação de Mestrado "Dimensionamento do pessoal de enfermagem das unidades de internação do Hospital São Paulo.", 2003 Universidade Federal de São Paulo - UNIFESP. E-mail: Mari@oftamo.epm.br Enfermeira, Mestre em Enfermagem pela UNIFESP.

Professora Titular do Departamento de Enfermagem da UNIFESP - Orientadora

3 Doutora em Estudos da Comunicação Humana. Professora Adjunto do Departamento de Enfermagem da UNIFESP .Co-orientadora
} 
visibilidade da realidade, e gerando maior complexidade para sua operacionalização ${ }^{(1)}$. Também o desconhecimento de enfermeiras acerca destas variáveis gera confrontos entre os objetivos por elas definidos, centrados nos valores humanos, com os objetivos da instituição hospitalar, que visa a eficácia e a eficiência da organização ${ }^{(1)}$.

O dimensionamento de pessoal de enfermagem é definido como sendo a:

etapa inicial do processo de provimento de pessoal, que tem por finalidade a previsão da quantidade de funcionários por categoria, requerida para suprir as necessidades de assistência de enfermagem, direta ou indiretamente prestada à clientela ${ }^{(4)}$.

Com fundamento nos dados apresentados na literatura sobre o tema, Gaidzinski ${ }^{(3)}$ caracterizou a evolução dos métodos utilizados para dimensionar o pessoal de enfermagem em quatro períodos, citados a seguir: o método intuitivo ou das relações de proporção (antes de 1939); introdução da variável horas médias de cuidado (1939); introdução das variáveis relativas às ausências (1947); introdução do Sistema de Classificação de Pacientes (1960) ${ }^{(1)}$.

Assim, verifica-se no Brasil, a partir de 1930, quando se iniciou a fase legal da administração de pessoal, que o método de cálculo de pessoal era fundamentado na proporção de trabalhadores necessários para realizar o número de tarefas a serem efetuadas diariamente, contando com dados subjetivos oriundos da vivência do administrador ${ }^{(1)}$. Este método, que não previa nenhum direito trabalhista, como folgas semanais remuneradas, férias, ou outras ausências, ainda tem sido utilizado em muitas instituições hospitalares brasileiras, por ser referendado por órgãos oficiais, como o Ministério da Saúde ${ }^{(5-6)}$.
No quarto período de evolução de dimensionamento de pessoal de enfermagem, os diferentes graus de complexidade assistencial passaram a ser considerados como instrumentos para o cálculo de estimativa de pessoal de enfermagem. No início da década de 80 do século XX, um trabalho apresentou um instrumento de classificação de pacientes estabelecendo o perfil de cada tipo de cuidado, a partir do conceito do Cuidado Progressivo ao Paciente, classificando os pacientes internados em quatro níveis de cuidado $^{(7)}$. Em 1994 foi desenvolvido um instrumento de classificação, estabelecendo cinco categorias de cuidados, de acordo com a complexidade assistencial dos pacientes internados: intensivos(I), semi-intensivos(S), alta dependência (AD), intermediários (IN) e mínimos (M) ${ }^{(8)}$.

A publicação da Resolução COFEN $n^{\circ} 189 / 96$ oficializou o cálculo de pessoal de enfermagem por meio de parâmetros para o quantitativo mínimo dos diferentes níveis de formação dos profissionais de enfermagem, recomendando que o dimensionamento do quadro de profissionais fundamente-se nas características referentes à instituição, ao serviço de enfermagem e à clientela $^{(9)}$.

A competência para o dimensionamento do pessoal de enfermagem é das enfermeiras que atuam diretamente na assistência, uma vez que identificam e avaliam continuamente os recursos existentes, face às necessidades da clientela assistida, podendo elaborar propostas adequadas para a prestação da assistência de enfermagem ${ }^{(10-11)}$.

Considerando as divergências existentes entre as chefias dos Serviços de Enfermagem e a Diretoria do Hospital São Paulo acerca do quantitativo do pessoal de enfermagem, foi desenvolvido este estudo visando dimensionar o pessoal de enfermagem segundo o modelo proposto por Gaidzinski ${ }^{(1)}$ e comparar os resultados obtidos com o quadro de pessoal de enfermagem dimensionado pela Diretoria de Enfermagem deste hospital.

\section{MÉTODO}

Este estudo do tipo descritivo foi realizado no Hospital São Paulo (HSP), cujas finalidades são a assistência, o ensino e a pesquisa.

A coleta de dados foi realizada após a aprovação do projeto de pesquisa pelo Comitê de Ética em Pesquisa da UNIFESP, seguindo as etapas recomendadas pelo modelo proposto por Gaidzinski ${ }^{(1)}$ :

- Conhecimento do perfil dos pacientes quanto à complexidade assistencial

A classificação dos pacientes foi realizada pelas enfermeiras das 40 unidades de internação, nos meses de março a maio de 2002, utilizando-se $\mathrm{o}$ instrumento de Fugulin et al. ${ }^{(8)}$.

- Levantamento do tempo de assistência de enfermagem

Para esta variável, foram utilizadas as horas médias de assistência de enfermagem preconizadas pela Resolução COFEN no ${ }^{\circ} 189 /$ $96^{(9)}$, acrescentando, para os pacientes que requerem cuidados de alta dependência, uma estimativa de seis horas, uma vez que esta Resolução não contempla este tipo de cuidado.

- Determinação do percentual de cada categoria profissional

Neste estudo foram considerados os percentuais recomendados por essa Resolução, acrescentandose os mesmos valores dos cuidados mínimos para os cuidados de alta dependência.

- Identificação da jornada efetiva de trabalho 
Considerando que os trabalhadores não são igualmente produtivos o tempo todo do turno de trabalho, optou-se, pelo percentual de $85,0 \%$ da jornada média de seis horas de trabalho, avaliado como excelente ${ }^{(12)}$.

- Identificação do percentual de ausências previstas e não previstas da equipe de enfermagem

As ausências previstas (dias destinados às folgas e férias), foram calculadas seguindo as equações do cálculo de pessoal ${ }^{(1)}$, adaptados às peculiaridades da instituição, como a inclusão dos feriados do ano nas folgas mensais.

As informações relativas às ausências não previstas (soma de diversas ausências, tais como faltas, licenças médicas, licenças maternidade, paternidade e nojo), foram coletas retrospectivamente, das escalas mensais de trabalho do período de janeiro a dezembro de 2001.

- Aplicação da equação para dimensionar o pessoal de enfermagem

O cálculo do pessoal de enfermagem foi realizado com a inserção dos dados coletados em planilha eletrônica, que facilita a operacionalização da equação, cedida pela Prof ${ }^{a}$. Dr. ${ }^{a}$ Raquel R. Gaidzinski e as etapas deste cálculo foram armazenadas neste banco de dados.

Os dados obtidos foram comparados com o quadro de dimensionamento de pessoal de enfermagem do HSP, que utiliza a equação da Associação Americana de Hospitais/ Liga Nacional de Educação de Enfermagem $^{(13)}$ com alguns ajustes. Desta forma, os dados utilizados para este cálculo são os utilizados desde 1998:
- Classificação de pacientes: calculada segundo o instrumento de Classificação de Pacientes de Fugulin et al. ${ }^{(8)}$, realizada apenas por um mês;

- Índice de Segurança Técnica: $35,0 \%$ (acordado com o Diretor Superintendente );

- Produtividade: 100\%;

- Carga horária semanal: 36 horas;

- Percentual de cada categoria profissional: $40,0 \%$ de enfermeiras e $60,0 \%$ de técnicos e auxiliares de enfermagem para cuidados intensivos; $30,0 \%$ de enfermeiras e $70,0 \%$ de técnicos e auxiliares de enfermagem para cuidados semiintensivos; $20,0 \%$ de enfermeiras e $80,0 \%$ de técnicos e auxiliares de enfermagem para cuidados de alta dependência, intermediários e mínimos.

\section{RESULTADOS E DISCUSSÃO}

\section{Classificação dos pacientes internados}

A admissão dos pacientes nas unidades de internação do HSP é realizada tendo em vista o ensino das disciplinas médicas, desconsiderando a complexidade assistencial. Esta situação produz distorções nos resultados do dimensionamento de pessoal e, dependendo do momento da coleta de dados referente a esta classificação, ocasiona a previsão maior ou menor do número de pessoal de enfermagem

No período da coleta de dados foram classificados 43.228 pacientes, sendo que o maior percentual destes $(30,8 \%)$ exigia nível de cuidados mínimos, apesar do HSP, ser categorizado de nível terciário e ser referência para a realização de procedimentos médicos de alta com- plexidade. A seguir, encontram-se os pacientes que demandaram cuidados intermediários $(27,5 \%)$, cuidados de alta dependência $(22,0 \%)$, cuidados intensivos $(12,0 \%) \mathrm{e}$, cuidados semiintensivos $(7,6 \%)$-Tabela 1.

$\mathrm{O}$ agrupamento de pacientes por sistemas de classificação da complexidade assistencial, além de melhorar a qualidade dos cuidados prestados, é viável também para alocar recursos humanos e materiais, diminuindo o custo dos cuidados de enfermagem ${ }^{(14)}$. A autora recomenda ainda que o instrumento de classificação seja utilizado como critério para a transferência dos pacientes.

Os critérios tradicionalmente adotados, em hospitais públicos e de ensino, distribuem os pacientes por especialidades médicas e sexo, dificultando tanto a prestação da assistência de enfermagem como a manutenção de recursos humanos e materiais para atender pacientes que requerem níveis de cuidados diversificados $^{(8)}$. Estes critérios ocasionam também situações de desconforto para pacientes e familiares, quando são obrigados a conviver com pacientes cujo estado geral encontra-se comprometido. A assistência de enfermagem nessa situação restringe-se ao atendimento das necessidades biológicas, em detrimento das psico-sócio-espirituais e educativas, comprometendo a integralidade dos cuidados prestados a esta clientela.

O instrumento de classificação utilizado mostrou-se inadequado para as unidades pediátricas, segundo as enfermeiras que realizaram a coleta de dados, por não caracterizarem o perfil desta clientela. 
Tabela 1 - Distribuição dos pacientes por unidades de internação, segundo níveis de complexidade dos cuidados de enfermagem, por número de leitos e número de dias. Hospital São Paulo, 2002.

\begin{tabular}{|c|c|c|c|c|c|c|c|c|}
\hline \multirow[t]{2}{*}{ Unidades de internaçăo } & \multirow{2}{*}{$\begin{array}{r}\text { No } \\
\text { Leitos }\end{array}$} & \multirow{2}{*}{$\begin{array}{c}\mathbf{N}^{\circ} \\
\text { Dias - }\end{array}$} & \multicolumn{6}{|c|}{$\begin{array}{l}\mathrm{N}^{\circ} \text { de pacientes segundo niveis de } \\
\text { complexidade }\end{array}$} \\
\hline & & & $\mathbf{M}$ & IN & AD & $\mathbf{S}$ & I & Total \\
\hline Ala $\mathrm{C} 08^{\circ}$ Andar & 12 & 92 & 17 & 216 & 575 & $=$ & 1 & 809 \\
\hline Ala C $07^{\circ}$ Andar & 10 & 91 & 15 & 358 & 359 & 19 & 19 & 770 \\
\hline Ala C $09^{\circ}$ Andar & 12 & 86 & 85 & 454 & 78 & 7 & - & 624 \\
\hline Ala C $11^{\circ}$ andar & 10 & 89 & 8 & 434 & 219 & 13 & $=$ & 674 \\
\hline $7^{\circ}$ Apartamentos & 2 & 91 & 28 & 35 & 50 & - & - & 113 \\
\hline Otorrino + Oftalmologia & 18 & 91 & 986 & 207 & - & - & - & 1193 \\
\hline Gastrocirurgia & 9 & 17 & 20 & 2 & 18 & - & $\cdot$ & 40 \\
\hline Gastrocirurgia & 24 & 89 & 621 & 311 & 416 & 21 & 1 & 1370 \\
\hline Ginecologia & 28 & 92 & 824 & 547 & 198 & 60 & - & 1629 \\
\hline Neurocirurgia & 28 & 88 & 391 & 376 & 849 & 122 & $=$ & 1738 \\
\hline Ortopedia & 38 & 89 & 399 & 718 & 878 & 138 & $=$ & 2133 \\
\hline Cirurgia Pediátrica & 9 & 89 & - & 102 & 448 & 106 & - & 656 \\
\hline Cirurgia Plástica & 32 & 88 & 1077 & 492 & 144 & 8 & - & 1721 \\
\hline Transplante Renal & 12 & 89 & 273 & 205 & 201 & 12 & 1 & 692 \\
\hline Urologia & 17 & 90 & 403 & 592 & 131 & 34 & 10 & 1170 \\
\hline Cirurgla Vascular & 15 & 89 & 307 & 387 & 380 & 4 & - & 1078 \\
\hline Cardiologia & 18 & 91 & 613 & 623 & 194 & 46 & - & 1476 \\
\hline Diálise & 11 & 92 & 368 & 113 & 242 & 2 & 133 & 858 \\
\hline Doenças Infecciosas Adulto (DIPA) & 18 & 89 & 216 & 282 & 87 & 486 & 153 & 1224 \\
\hline Gastroclínica & 14 & 92 & 561 & 98 & 54 & 95 & 8 & 816 \\
\hline Geriatria/endocrino/Tórax & 16 & 89 & 748 & 20 & 343 & 3 & - & 1114 \\
\hline Hematologia+Otorrinopediatria & 15 & 89 & 271 & 656 & 161 & 11 & 4 & 1103 \\
\hline Clinica Médica Feminina & 22 & 89 & 281 & 752 & 425 & 19 & 4 & 1481 \\
\hline Clinica Médica Masculina & 21 & 89 & 43 & 745 & 458 & 37 & 1 & 1284 \\
\hline Neuroclínica & 12 & 88 & 448 & 162 & 226 & 29 & 32 & 897 \\
\hline Pneumologia & 12 & 89 & 569 & 124 & 138 & - & 3 & 834 \\
\hline Psiquiatria & 18 & 81 & 857 & 57 & 36 & - & - & 950 \\
\hline Obstetricia & 29 & 89 & 1131 & 777 & 95 & - & - & 2003 \\
\hline Berçário & 39 & 89 & - & 44 & 276 & 505 & 1020 & 1845 \\
\hline Doenças Infecciosas Pediátrica (DIPe) & 11 & 89 & 663 & 153 & 89 & 6 & 1 & 912 \\
\hline Pediatria & 32 & 92 & 154 & 580 & 1254 & 425 & 112 & 2525 \\
\hline Retaguarda P.S. crônica & 2 & 28 & 7 & 2 & 10 & 5 & - & 24 \\
\hline Retaguarda P.S. crônica & 18 & 90 & 712 & 659 & 199 & 36 & - & 1606 \\
\hline Retaguarda P.S. semi & 11 & 89 & 226 & 408 & 214 & 83 & 37 & 968 \\
\hline UTI Cardiologia & 8 & 90 & - & 32 & - & 295 & 350 & 677 \\
\hline UTI Clínica Médica & 5 & 89 & - & - & $=$ & 63 & 355 & 418 \\
\hline UTI Geral & 16 & 89 & - & - & 1 & 268 & 916 & 1185 \\
\hline UTI Neurocirurgia & 5 & 89 & - & - & 5 & 47 & 360 & 412 \\
\hline UTI Pediátrica & 9 & 89 & - & - & 16 & 143 & 478 & 637 \\
\hline UTI Pneumologia & 5 & 89 & - & 1 & 35 & 6 & 285 & 327 \\
\hline UTI Retaguarda grave & 9 & 89 & 5 & 159 & 37 & 104 & 489 & 794 \\
\hline $\begin{array}{l}\text { Unidade de Pós-operatório cirurgia } \\
\text { cardíaca (UPOCC) }\end{array}$ & 6 & 88 & - & - & - & 19 & 429 & 448 \\
\hline Total & 658 & & $\begin{array}{r}13327 \\
30,8 \\
\end{array}$ & $\begin{array}{r}11883 \\
27,5 \\
\end{array}$ & $\begin{array}{r}9539 \\
22,0\end{array}$ & $\begin{array}{r}3277 \\
7,6 \\
\end{array}$ & $\begin{array}{r}5202 \\
12,0\end{array}$ & $\begin{array}{l}43228 \\
100,0\end{array}$ \\
\hline
\end{tabular}

Levantamento do tempo de assistência de enfermagem

Os dados sobre as médias de horas de enfermagem despendidas por paciente/dia, segundo os níveis de complexidade assistencial, são demonstrados na Tabela 2.
Apesar da classificação dos pacientes demonstrar que aqueles que requeriam cuidados mínimos constituíram a maioria dos internados nas unidades, verifica-se na Tabela 2 que os pacientes de cuidados intensivos demandaram maior tempo de assistência prestada pela equipe de enfermagem (30,9\%), seguidos pelos de cuidados de alta dependência $(21,8 \%)$, cuidados intermediários $(21,2 \%)$, cuidados mínimos $(15,3 \%)$ e cuidados semi-intensivos $(10,7 \%)$. 
Tabela 2 - Médias e percentagem de horas de cuidados de enfermagem por pacientes/dia, segundo grupos de unidades de internação por níveis de complexidade assistencial. (Hospital São Paulo, 2002)

\begin{tabular}{|c|c|c|c|c|c|c|c|}
\hline \multirow{2}{*}{ Grupos de unidades } & & \multicolumn{5}{|c|}{ Niveis de complexidade } & \multirow{2}{*}{$\begin{array}{c}\text { Total } \\
\%\end{array}$} \\
\hline & & $M$ & IN & AD & $\mathbf{s}$ & $\mathbf{I}$ & \\
\hline \multirow{2}{*}{ Cirúrgicas (Cir.) } & $\bar{x}$ & 180,3 & 216,3 & 252,3 & 48,1 & 2,1 & 699,1 \\
\hline & $\%$ & 25,8 & 30,9 & 36,1 & 6,9 & 0,3 & 100,0 \\
\hline \multirow{2}{*}{ Clínico-cirúrgicas } & $\overline{\bar{x}}$ & 62,5 & 142,2 & 137,6 & 16,8 & 9,8 & 369,0 \\
\hline & $\%$ & 16,9 & 38,5 & 37,3 & 4,6 & 2,7 & 100,0 \\
\hline \multirow{2}{*}{ Clínicas (Cl.) } & $\overline{\bar{x}}$ & 134,9 & 162,0 & 124,9 & 67,8 & 57,1 & 546,7 \\
\hline & $\%$ & 24,7 & 29,6 & 22,8 & 12,4 & 10,4 & 100,0 \\
\hline \multirow{2}{*}{ Cuidados intensivos } & $\bar{x}$ & 0,2 & 10,6 & 6,3 & 90,0 & 633,8 & 740,8 \\
\hline & $\%$ & 0,0 & 1,4 & 0,9 & 12,1 & 85,6 & 100,0 \\
\hline \multirow{2}{*}{ Obstétricas } & $\overline{\bar{x}}$ & 38,1 & 42,8 & 6,4 & - & - & 87,3 \\
\hline & $\%$ & 43,6 & 49,0 & 7,3 & - & - & 100,0 \\
\hline \multirow{2}{*}{ Pediátricas (Ped.) } & $\overline{\bar{x}}$ & 27,4 & 41,7 & 106,4 & 88,1 & 195,4 & 459,0 \\
\hline & $\%$ & 6,0 & 9,1 & 23,2 & 19,2 & 42,6 & 100,0 \\
\hline \multirow{2}{*}{ Total } & $\bar{x}$ & 443,4 & 615,6 & 633,9 & 310,8 & 898,1 & $2.901,9$ \\
\hline & $\%$ & 15,3 & 21,2 & 21,8 & 10,7 & 30,9 & 100,0 \\
\hline
\end{tabular}

Ausências previstas e não previstas da equipe de enfermagem

Os dados relacionados às ausências previstas e não previstas e o total de acréscimo referentes às enfermeiras e aos profissionais de nível médio são apresentados nas Tabelas 3 e 4.

Pode-se observar, na Tabela 3, que as unidades que apresentaram um percentual maior de ausências não previstas das enfermeiras foram: Ala $\mathrm{C}$ do $9^{\circ}$ andar $(17,0 \%)$; Cirurgia Plástica (16,4\%); UPOCC $(15,7 \%)$ e Pediatria $(11,4 \%)$. Este resultado foi devido às licenças maternidade e às licenças prolongadas por perícia médica, e indica que provavelmente as licenças médicas não estejam associadas ao ambiente de trabalho, uma vez que apenas uma das unidades ( UPOCC) é de terapia intensiva, onde se supõe que o trabalho seja mais estressante e com maior risco ocupacional.

Analisando os motivos que levam os trabalhadores de enfermagem ao absenteísmo, um estudo ressalta que não se pode tratar os dados meramente sob o ponto de vista quantitativo, mas também sob a óptica qualitativa, considerando os trabalhadores como pessoas que têm vínculos fora da instituição ${ }^{(15)}$. As folgas representaram um percentual maior das ausências, sendo que os valores de $44,8 \%$, da Otorrino+Oftalmologia e $45,4 \%$, da Retaguarda do Pronto Socorro Crônicos, foram atribuídos ao maior número de enfermeiras com vínculo UNIFESP, que tiveram dez folgas por mês, em 2001. A necessidade de acréscimo mais expres- siva foi a da UPOCC $(76,0 \%)$, seguida da unidade de Cirurgia Plástica $(73,0 \%)$.

Quanto aos profissionais de nível médio, observa-se na Tabela 4 que a unidade de internação da $\mathrm{Ci}$ rurgia Plástica apresentou o maior percentual de ausências não previstas $(12,4 \%)$, devido aos funcionários em perícia médica. As folgas representaram o maior percentual das ausências, com valores de $38,3 \%$, encontrados nas unidades de: Neurocirurgia; Cirurgia Pediátrica; Gastroclínica; Geriatria+ Endócrino+Tórax; Berçário; DIPe; UTI Cardiologia; UTI Geral e UTI Neurocirurgia. O percentual de total da necessidade de acréscimo mais significativo foi de $62,0 \%$, nas unidades de: Neurocirurgia; Cirurgia Plástica e UTI Neurocirurgia. 
Tabela 3 - Ausências previstas e não previstas das enfermeiras, segundo média de pessoal e total de acréscimo, por unidades de internação. Hospital São Paulo, 2001.

\begin{tabular}{|c|c|c|c|c|c|}
\hline \multirow{4}{*}{ Unidades de internaçāo } & \multirow{3}{*}{$\begin{array}{c}\text { Média } \\
\text { de } \\
\text { Pessoal }\end{array}$} & \multicolumn{3}{|c|}{ Ausências } & \multirow{4}{*}{$\begin{array}{r}\begin{array}{r}\text { Total de } \\
\text { acréscimo }\end{array} \\
\%\end{array}$} \\
\hline & & \multirow{2}{*}{$\begin{array}{c}\text { Näo } \\
\text { Previstas }\end{array}$} & \multicolumn{2}{|c|}{ Previstas } & \\
\hline & & & Férias & Folgas & \\
\hline & $\mathrm{x}$ & & $\%$ & $\%$ & \\
\hline Ala C $08^{\circ}$ Andar & 5,5 & 7,3 & 9,0 & 32,2 & 55,0 \\
\hline Ala C $07^{\circ}$ Andar & 4,1 & 1,7 & 9,0 & 32,2 & 47,0 \\
\hline Ala C 09o Andar & 3,9 & 17,0 & 9,0 & 32,2 & 69,0 \\
\hline Ala C $11^{\circ}$ Andar & 3,8 & 4,0 & 9,0 & 32,2 & 50,0 \\
\hline $7^{\circ}$ Apartamentos & - & - & - & - & \\
\hline Otorrino + Oftalmologia & 1,4 & 6,9 & 9,0 & 44,8 & 69,0 \\
\hline Gastrocirurgia & 4,3 & 1,0 & 9,0 & 32,2 & 45,0 \\
\hline Ginecologia & 4,0 & 9,0 & 9,0 & 35,2 & 61,0 \\
\hline Neurocirurgia & 10,3 & 3,3 & 9,0 & 39,3 & 57,0 \\
\hline Ortopedia & 5,5 & 0,7 & 9,0 & 32,2 & 45,0 \\
\hline Cirurgia Pediátrica & 4,0 & 0,8 & 9,0 & 38,3 & 52,0 \\
\hline Cirurgia Plástica & 3,2 & 16,4 & 9,0 & 36,2 & 73,0 \\
\hline Transplante Renal & 2,8 & 1,3 & 9,0 & 36,2 & 50,0 \\
\hline Urologia & 6,6 & 0,5 & 9,0 & 32,2 & 45,0 \\
\hline Cirurgia Vascular & 6,6 & 0,5 & 9,0 & 32,2 & 45,0 \\
\hline Cardiologia & 8,5 & 1,3 & 9,0 & 32,2 & 46,0 \\
\hline Diálise & 10,2 & 1,4 & 9,0 & 38,3 & 53,0 \\
\hline Doenças Infecciosas Adulto (DIPA) & 7,0 & 1,4 & 9,0 & 38,3 & 53,0 \\
\hline Gastroclinica & 3,9 & 1,2 & 9,0 & 41,5 & 56,0 \\
\hline Geriatria/Endocrino/Tórax & 4,0 & 0,8 & 9,0 & 38,3 & 52,0 \\
\hline Hematologia+otorrinopediatria & 3,8 & 9,9 & 9,0 & 32,2 & 58,0 \\
\hline Clínica Médica Feminina & 3,3 & 2,9 & 9,0 & 36,2 & 53,0 \\
\hline Clínica Médica Masculina & 6,3 & 3,2 & 9,0 & 38,3 & 55,0 \\
\hline Neuroclínica & 4,3 & 10,0 & 9,0 & 35,2 & 62,0 \\
\hline Pneumologia & 11,3 & 4,7 & 9,0 & 38,3 & 58,0 \\
\hline Psiquiatria & 1,1 & 6,0 & 9,0 & 32,2 & 53,0 \\
\hline Obstetricia & 6,1 & 2,6 & 9,0 & 38,3 & 55,0 \\
\hline Berçário & 13,4 & 4,0 & 9,0 & 41,5 & 60,0 \\
\hline Doenças Infecciosas Pediátrica (DIPe) & 3,7 & 1,0 & 9,0 & 41,5 & 56,0 \\
\hline Pediatria & 10,6 & 11,4 & 9,0 & 35,7 & 65,0 \\
\hline Retaguarda do P.S.crônica & 6,8 & 5,5 & 9,0 & 45,4 & 67,0 \\
\hline Retaguarda do P.S. semi & 5,4 & 4,3 & 9,0 & 38,3 & 57,0 \\
\hline UTI Cardiologia & 8,3 & 4,2 & 9,0 & 38,3 & 57,0 \\
\hline UTI Clinica Médica & 6,3 & 3,2 & 9,0 & 38,3 & 55,0 \\
\hline UTI Geral & 19,4 & 2,8 & 9,0 & 38,3 & 55,0 \\
\hline UTI Neurocirurgia & 10,3 & 3,3 & 9,0 & 39,3 & 57,0 \\
\hline UTI Pediátrica & 12,0 & 3,8 & 9,0 & 39,3 & 58,0 \\
\hline UTI Pneumologia & 11,3 & 4,7 & 9,0 & 32,2 & 58,0 \\
\hline Retaguarda P.S. grave & 8,8 & 3,2 & 9,0 & 39,3 & 57,0 \\
\hline $\begin{array}{l}\text { Unidade de Pós-operatório cirurgia } \\
\text { cardíaca (UPOCC) }\end{array}$ & 8,5 & 15,7 & 9,0 & 39,8 & 76,0 \\
\hline
\end{tabular}


Tabela 4 - Ausências previstas e não previstas dos técnicos/auxiliares de enfermagem, segundo média de pessoal e total de acréscimo por unidades de internação. Hospital São Paulo, 2001.

\begin{tabular}{|c|c|c|c|c|c|}
\hline \multirow{4}{*}{ Unidades de internaçăo } & \multirow{3}{*}{$\begin{array}{l}\text { Média de } \\
\text { Pessoal }\end{array}$} & \multicolumn{3}{|c|}{ Ausências } & \multirow{4}{*}{$\begin{array}{r}\text { Total de } \\
\text { acréscimo } \\
\%\end{array}$} \\
\hline & & \multirow{2}{*}{$\begin{array}{c}\text { Não } \\
\text { Previstas }\end{array}$} & \multicolumn{2}{|c|}{ Previstas } & \\
\hline & & & Férias & Folgas & \\
\hline & $\mathrm{x}$ & $\%$ & $\%$ & $\%$ & \\
\hline $\begin{array}{l}\text { Ala C } 08^{\circ} \text { Andar } \\
\text { Ala C } 07^{\circ} \text { Andar }\end{array}$ & 20,4 & 2,1 & 9,0 & 32,2 & 47,0 \\
\hline $\begin{array}{l}\text { Ala C } 07^{\circ} \text { Andar } \\
\text { Ala C } 09^{\circ} \text { Andar }\end{array}$ & 15,2 & 1,5 & 9,0 & 32,2 & 47,0 \\
\hline $\begin{array}{l}\text { Ala C } 09^{\circ} \text { Andar } \\
\text { Ala C } 11^{\circ} \text { Andar }\end{array}$ & 15,9 & 4,6 & 9,0 & 32,2 & 51,0 \\
\hline $\begin{array}{l}\text { Ala C } 11^{\circ} \text { Andar } \\
7^{\circ} \text { Apartamentos }\end{array}$ & 14,6 & 7,3 & 9,0 & 32,2 & 55,0 \\
\hline $\begin{array}{l}7^{0} \text { Apartamentos } \\
\text { Otorrino+Oftalmologia }\end{array}$ & 4,2 & 2,1 & 9,0 & 32,2 & 47,0 \\
\hline $\begin{array}{l}\text { Otorrino+Oftalmologia } \\
\text { Gastrocirurgia }\end{array}$ & 12,4 & 5,9 & 9,0 & 32,2 & 53,0 \\
\hline $\begin{array}{l}\text { Gastrocirurgia } \\
\text { Ginecologia }\end{array}$ & 16,8 & 2,4 & 9,0 & 32,2 & 48,0 \\
\hline $\begin{array}{l}\text { Ginecologia } \\
\text { Neurocirurgia }\end{array}$ & 13,0 & 1,7 & 9,0 & 32,2 & 47,0 \\
\hline $\begin{array}{l}\text { Neurocirurgia } \\
\text { Ortopedia }\end{array}$ & 29,5 & 7,5 & 9,0 & 38,3 & 62,0 \\
\hline $\begin{array}{l}\text { Ortopedia } \\
\text { Cirurgia Pediátrica }\end{array}$ & 19,5 & 5,7 & 9,0 & 32,2 & 52,0 \\
\hline $\begin{array}{l}\text { Cirurgia Pediátrica } \\
\text { Cirurgia Plástica }\end{array}$ & 6,8 & 0,7 & 9,0 & 38,3 & 52,0 \\
\hline $\begin{array}{l}\text { Cirurgia Plástica } \\
\text { Transplante Renal }\end{array}$ & 13,9 & 12,4 & 9,0 & 32,2 & 62,0 \\
\hline $\begin{array}{l}\text { Transplante Renal } \\
\text { Urologia }\end{array}$ & 12,5 & 3,6 & 9,0 & 32,2 & 49,0 \\
\hline $\begin{array}{l}\text { Urologia } \\
\text { Cirurgia Vascular }\end{array}$ & 23,5 & 3,5 & 9,0 & 32,2 & 49,0 \\
\hline $\begin{array}{l}\text { Cirurgia Vascular } \\
\text { Cardiologia }\end{array}$ & 23,5 & 3,5 & 9,0 & 32,2 & 49,0 \\
\hline $\begin{array}{l}\text { Cardiologia } \\
\text { Diállise }\end{array}$ & 16,9 & 8,0 & 9,0 & 32,2 & 56,0 \\
\hline $\begin{array}{l}\text { Diálise } \\
\text { Doenças Infecciosas Adulto (DIPA) }\end{array}$ & 29,1 & 3,0 & 9,0 & 32,2 & 48,0 \\
\hline $\begin{array}{l}\text { Doenças Infecciosas Adulto (DIPA) } \\
\text { Gastroclinica }\end{array}$ & 22,0 & 6,9 & 9,0 & 32,2 & 54,0 \\
\hline $\begin{array}{l}\text { Gastroclínica } \\
\text { Geriatria/Endocrino/Torax }\end{array}$ & 13,1 & 1,6 & 9,0 & 38,3 & 53,0 \\
\hline $\begin{array}{l}\text { Geriatria/Endocrino/Torax } \\
\text { Hematologia+otorrinopediatria }\end{array}$ & 13,8 & 4,3 & 9,0 & 38,3 & 57,0 \\
\hline $\begin{array}{l}\text { Hematologia+otorrinopediatria } \\
\text { Clínica Médica Feminina }\end{array}$ & 15,3 & 4,0 & 9,0 & 32,2 & 50,0 \\
\hline $\begin{array}{l}\text { Clínica Médica Feminina } \\
\text { Clínica Médica Masculina }\end{array}$ & 15,6 & 3,4 & 9,0 & 32,2 & 49,0 \\
\hline $\begin{array}{l}\text { Clínica Médica Masculina } \\
\text { Neuroclínica }\end{array}$ & 26,3 & 4,6 & 9,0 & 32,2 & 51,0 \\
\hline $\begin{array}{l}\text { Neuroclínica } \\
\text { Pneumologia }\end{array}$ & 13,4 & 2,1 & 9,0 & 32,2 & 47,0 \\
\hline $\begin{array}{l}\text { Pneumologia } \\
\text { Psiquiatria }\end{array}$ & 32,5 & 2,4 & 9,0 & 32,2 & 48,0 \\
\hline $\begin{array}{l}\text { Psiquiatria } \\
\text { Obstetricia }\end{array}$ & 12,3 & 3,9 & 9,0 & 32,2 & 50,0 \\
\hline $\begin{array}{l}\text { Obstetricia } \\
\text { Berçário }\end{array}$ & 15,3 & 7,7 & 9,0 & 32,2 & 55,0 \\
\hline $\begin{array}{l}\text { Berçário } \\
\text { Doenças Infecciosas Pediátrica(DIPe) }\end{array}$ & 45,3 & 4,5 & 9,0 & 38,3 & 57,0 \\
\hline $\begin{array}{l}\text { Doenças Infecciosas Pediátrica(DIPe) } \\
\text { Pediatria }\end{array}$ & 12,6 & 4,3 & 9,0 & 38,3 & 57,0 \\
\hline $\begin{array}{l}\text { Pediatria } \\
\text { Retaguarda PS crónica }\end{array}$ & 36,0 & 1,2 & 9,0 & 32,2 & 46,0 \\
\hline $\begin{array}{l}\text { Retaguarda PS crónica } \\
\text { Retaguarda PS semi }\end{array}$ & 27,1 & 6,5 & 9,0 & 32,2 & 53,0 \\
\hline $\begin{array}{l}\text { Retaguarda PS semi } \\
\text { UTI Cardiologia }\end{array}$ & 25,2 & 5,6 & $\begin{array}{l}9,0 \\
9,0\end{array}$ & $\begin{array}{l}32,2 \\
38,3\end{array}$ & 52,0 \\
\hline $\begin{array}{l}\text { UTI Cardiologia } \\
\text { UTI Clinica Médica }\end{array}$ & 16,6 & 5,1 & $\begin{array}{l}9,0 \\
9,0\end{array}$ & $\begin{array}{l}38,3 \\
32,2\end{array}$ & $\begin{array}{l}58,0 \\
51,0\end{array}$ \\
\hline $\begin{array}{l}\text { UTI Clinica Médica } \\
\text { UTI Geral }\end{array}$ & $\begin{array}{l}26,3 \\
50,0\end{array}$ & $\begin{array}{l}4,6 \\
4,7\end{array}$ & $\begin{array}{l}9,0 \\
9,0\end{array}$ & $\begin{array}{l}32,2 \\
38,3\end{array}$ & $\begin{array}{l}51,0 \\
58,0\end{array}$ \\
\hline $\begin{array}{l}\text { UTI Geral } \\
\text { UTI Neurocirurgia }\end{array}$ & $\begin{array}{l}50,0 \\
29,5\end{array}$ & 7,5 & 9,0 & 38,3 & $\begin{array}{l}58,0 \\
62,0\end{array}$ \\
\hline UTI Pediátrica & 61,6 & 0,8 & 9,0 & 32,2 & 45,0 \\
\hline UTI Pneumologia & 32,5 & 2,4 & 9,0 & 32,2 & 48,0 \\
\hline Retaguarda PS grave & 28,3 & 3,2 & 9,0 & 32,2 & 49,0 \\
\hline $\begin{array}{l}\text { Unidade de Pós-operatório cirurgia } \\
\text { cardiaca (UPOCC) }\end{array}$ & 15,7 & 3,9 & 9,0 & 32,2 & 50,0 \\
\hline
\end{tabular}

Observa-se nas Tabelas 3 e 4 que as ausências previstas representaram um percentual maior que o de ausências não previstas, nas categorias de enfermeiras e de técnicos/ auxiliares de enfermagem, sendo que os valores mais expressivos foram os referentes às folgas. Esta proporção elevada reflete a política de Re- cursos Humanos da UNIFESP, freqüentemente estendida aos funcionários contratados pelo HSP, de compensar os baixos salários com um maior número de folgas, e até mesmo diferenciar as unidades de internação consideradas "fechadas" ou insalubres com uma folga a mais.
Esta medida gera a necessidade de maior acréscimo de funcionários para a cobertura das ausências.

A Tabela 5 apresenta o quadro de pessoal atual e o projetado resultante da aplicação do modelo de Gaidzinski(1), segundo as unidades de internação. 
Tabela 5 - Quadros de profissionais de enfermagem existente e projetado, por número de leitos e relação leitos por enfermeiras e técnicos/auxiliares de enfermagem do quadro projetado, segundo unidades de internação. (Hospital São Paulo, 2002)

\begin{tabular}{|c|c|c|c|c|c|c|c|}
\hline \multirow{4}{*}{ Unidades de internação } & \multicolumn{4}{|c|}{ Quadro de Pessoal } & \multirow{4}{*}{$\begin{array}{r}\text { Leitos } \\
\mathbf{N}^{\circ}\end{array}$} & \multirow{3}{*}{\multicolumn{2}{|c|}{$\begin{array}{l}\text { Leitos por } \\
\\
\text { Enf } \begin{array}{r}\text { Tec/ } \\
\text { Aux }\end{array}\end{array}$}} \\
\hline & \multicolumn{2}{|c|}{ Existente } & \multicolumn{2}{|c|}{ Projetado } & & & \\
\hline & Enf & $\begin{array}{r}\text { Tec/ } \\
\text { Aux }\end{array}$ & Enf & $\begin{array}{c}\text { Tec/ } \\
\text { Aux }\end{array}$ & & & \\
\hline & $\mathbf{N}^{\circ}$ & $\mathrm{N}^{\circ}$ & $\mathbf{N}^{\circ}$ & $\mathrm{N}^{\circ}$ & & & \\
\hline Ala C $08^{\circ}$ Andar & 4 & 14 & 4 & 10 & 12 & 3,0 & 1,2 \\
\hline Ala C $07^{\circ}$ Andar & 4 & 10 & 4 & 10 & 10 & 2,5 & 1,0 \\
\hline Ala C $09^{\circ}$ Andar & 4 & 10 & 3 & 8 & 12 & 4,0 & 1,5 \\
\hline Ala C $11^{\circ}$ Andar & 4 & 10 & 3 & 9 & 10 & 3,3 & 1,1 \\
\hline Ala C Apto $7^{\circ}$ & 1 & 4 & - & 1 & 2 & - & 2,0 \\
\hline Otorrino+Oftalmologia & 2 & 11 & 4 & 10 & 18 & 4,5 & 1,8 \\
\hline Gastrocirurgia & 7 & 17 & 6 & 16 & 33 & 5,5 & 2,0 \\
\hline Ginecologia & 4 & 11 & 7 & 15 & 28 & 4,0 & 1,8 \\
\hline Neurocirurgia & 6 & 18 & 9 & 24 & 28 & 3,1 & 1,1 \\
\hline Ortopedia & 6 & 20 & 10 & 27 & 38 & 3,8 & 1,4 \\
\hline Cirurgia Pediátrica & 4 & 8 & 4 & 10 & 9 & 2,2 & 0,9 \\
\hline Cirurgia Plástica & 4 & 13 & 7 & 17 & 32 & 4,5 & 1,8 \\
\hline Transplante Renal & 5 & 10 & 3 & 8 & 12 & 4,0 & 1,5 \\
\hline Urologia & 4 & 14 & 5 & 12 & 17 & 3,4 & 1,4 \\
\hline Cirurgia Vascular & 4 & 13 & 4 & 12 & 15 & 3,7 & 1,2 \\
\hline Cardiologia & 4 & 10 & 6 & 16 & 18 & 3,0 & 1,1 \\
\hline Diálise & 5 & 12 & 6 & 10 & 11 & 1,8 & 1,1 \\
\hline Doenças Infecciosas Adulto (DIPA) & 9 & 19 & 12 & 18 & 18 & 1,5 & 1,0 \\
\hline Gastroclínica & 4 & 12 & 4 & 8 & 14 & 3,5 & 1,7 \\
\hline Geriatria/Endocrino/Tórax & 4 & 13 & 4 & 11 & 16 & 4,0 & 1,4 \\
\hline Hematologia+Otorrinopediatria & 4 & 13 & 5 & 12 & 15 & 3,0 & 1,2 \\
\hline Clínica Médica Feminina & 4 & 16 & 7 & 17 & 22 & 3,1 & 1,2 \\
\hline Clínica Médica Masculina & 7 & 25 & 6 & 16 & 21 & 3,5 & 1,3 \\
\hline Neuroclínica & 4 & 14 & 5 & 10 & 12 & 2,4 & 1,2 \\
\hline Pneumologia & 4 & 10 & 3 & 8 & 12 & 4,0 & 1,5 \\
\hline Psiquiatria & 2 & 12 & 3 & 8 & 18 & 6,0 & 2,2 \\
\hline Obstetricia & 8 & 18 & 7 & 19 & 29 & 4,1 & 1,5 \\
\hline Berçário & 20 & 47 & 39 & 38 & 39 & 1,0 & 1,0 \\
\hline Doenças Infecciosas Pediátrica(DIPe) & 4 & 10 & 3 & 8 & 11 & 3,6 & 1,3 \\
\hline Pediatria & 10 & 32 & 19 & 34 & 32 & 1,6 & 0,9 \\
\hline Retaguarda PS crônica & 8 & 28 & 7 & 18 & 20 & 2,8 & 1,1 \\
\hline Retaguarda PS semi & 8 & 22 & 6 & 12 & 11 & 1,8 & 0,9 \\
\hline UTI Cardiologia & 9 & 16 & 14 & 14 & B & 0,5 & 0,5 \\
\hline UTI Clínica Médica & 8 & 8 & 11 & 9 & 5 & 0,4 & 0,5 \\
\hline UTI Geral & 24 & 41 & 30 & 27 & 16 & 0,5 & 0,5 \\
\hline UTI Neurocirurgia & 8 & 12 & 11 & 10 & 5 & 0,4 & 0,5 \\
\hline UTI Pediátrica & 15 & 24 & 16 & 13 & 9 & 0,5 & 0,6 \\
\hline UTI Pneumologia & 6 & 14 & 8 & 7 & 5 & 0,6 & 0,7 \\
\hline Retaguarda PS grave & 10 & 28 & 17 & 15 & 9 & 0,5 & 0,6 \\
\hline $\begin{array}{l}\text { Unidade de Pós-operatório cirurgia } \\
\text { cardiaca (UPOCC) }\end{array}$ & 9 & 15 & 15 & 10 & 6 & 0,4 & 0,6 \\
\hline Total & 261 & 654 & 337 & 557 & 658 & 0,5 & 0,8 \\
\hline
\end{tabular}

Na Tabela 6 estão apresentados os mesmos quadros de pessoal da Tabela 5, distribuídos segundo os grupos de unidades de internação.
A comparação entre os quadros de pessoal de enfermagem existente e projetado, nas Tabelas 5 e 6 , mos- tra discrepância entre o número de enfermeiras e dos técnicos/auxiliares de enfermagem. 
Tabela 6 - Quadros de profissionais de enfermagem existente e projetado, por número de leitos e relação de leitos por enfermeiras e técnicos/auxiliares de enfermagem do quadro projetado, segundo grupos de unidades de internação. (Hospital São Paulo, 2002)

\begin{tabular}{|c|c|c|c|c|c|c|c|}
\hline \multirow{4}{*}{ Grupos de unidades } & \multicolumn{4}{|c|}{ Quadro de Pessoal } & \multirow{4}{*}{$\frac{\text { Leitos }}{\mathbf{N}^{0}}$} & \multirow{2}{*}{\multicolumn{2}{|c|}{ Leitos por }} \\
\hline & \multicolumn{2}{|c|}{ Existente } & \multicolumn{2}{|c|}{ Projetado } & & & \\
\hline & Enf & $\begin{array}{c}\text { Tec/ } \\
\text { Aux }\end{array}$ & Enf & $\begin{array}{c}\text { Tec/ } \\
\text { Aux }\end{array}$ & & \multirow[t]{2}{*}{ Enf } & \multirow{2}{*}{$\begin{array}{r}\text { Tec/ } \\
\text { Aux }\end{array}$} \\
\hline & No & $\mathrm{N}^{\circ}$ & $\mathbf{N}^{\circ}$ & $\mathrm{N}^{\circ}$ & & & \\
\hline Cirúrgicas (Cir.) & $\begin{array}{r}46 \\
(25,4) \\
\end{array}$ & $\begin{array}{r}135 \\
(74,6) \\
\end{array}$ & $\begin{array}{r}59 \\
(28,1) \\
\end{array}$ & $\begin{array}{r}151 \\
(71,9)\end{array}$ & 230 & 3,9 & 1,5 \\
\hline Clínico-cirúrgicas & $\begin{array}{r}37 \\
(25,0) \\
\end{array}$ & $\begin{array}{r}111 \\
(75,0)\end{array}$ & $\begin{array}{r}31 \\
(28,2) \\
\end{array}$ & $\begin{array}{r}79 \\
(71,8)\end{array}$ & 93 & 3,0 & 1,2 \\
\hline Clínicas (Cl.) & $\begin{array}{r}47 \\
(25,2) \\
\end{array}$ & $\begin{array}{r}143 \\
(74,8) \\
\end{array}$ & $\begin{array}{r}57 \\
(31,7) \\
\end{array}$ & $\begin{array}{r}123 \\
(68,3) \\
\end{array}$ & 161 & 2,8 & 1,3 \\
\hline Cuidados intensivos & $\begin{array}{r}89 \\
(36,0) \\
\end{array}$ & $\begin{array}{r}158 \\
(64,0) \\
\end{array}$ & $\begin{array}{r}122 \\
(53,7)\end{array}$ & $\begin{array}{r}105 \\
(46,3)\end{array}$ & 63 & 0,5 & 0,6 \\
\hline Obstétrica & $\begin{array}{r}8 \\
(30,8) \\
\end{array}$ & $\begin{array}{r}18 \\
(69,2)\end{array}$ & $\begin{array}{r}7 \\
(26,9)\end{array}$ & $\begin{array}{r}19 \\
(73,1)\end{array}$ & 29 & 4,1 & 1,5 \\
\hline Pediátricas (Ped.) & $\begin{array}{r}34 \\
(27,6) \\
\end{array}$ & $\begin{array}{r}89 \\
(72,4)\end{array}$ & $\begin{array}{r}61 \\
(43,3)\end{array}$ & $\begin{array}{r}80 \\
(56,7) \\
\end{array}$ & 82 & 1,3 & 1,0 \\
\hline Total & $\begin{array}{r}261 \\
(28,6)\end{array}$ & $\frac{654}{(71,4)}$ & $\begin{array}{r}337 \\
(37,7) \\
\end{array}$ & $\begin{array}{r}557 \\
(62,3)\end{array}$ & 658 & 1,9 & 1,1 \\
\hline
\end{tabular}

Quanto às enfermeiras, o número projetado é maior do que o quadro existente nas unidades cirúrgicas, clínicas, de cuidados intensivos e pediátricas. Em relação aos profissionais de nível médio, o quadro atual é maior do que o projetado em quatro grupos de unidades, principalmente nas UTIs e clínicas. Pelos cálculos efetuados segundo o modelo Gaidzinski ${ }^{1}$, seria necessário admitir mais 76 enfermeiras e reduzir em 97 o número de técnicos/auxiliares de enfermagem do quadro dimensionado pela Diretoria de Enfermagem do HSP.

Quanto à relação número de leitos por enfermeiras do quadro projetado, observa-se na Tabela 5 que, quando analisada por unidades de internação, a menor foi para a UPOCC $(0,4)$ e a maior para a Psiquiatria $(6,0)$. Para os técnicos/auxiliares de enfermagem, a menor relação foi na UTI Neurocirurgia $(0,5)$ e a maior para a unidade da Psiquiatria $(2,2)$.

Na Tabela 6, a distribuição por grupos de unidades permite verificar que a menor relação encontrada foi para as UTIs $(0,5$ leitos/enfer- meiras e 0,6 leitos por técnicos/auxiliares de enfermagem), e a maior foi para a unidade obstétrica $(4,1$ leitos/enfermeiras e 1,5 leitos/ técnicos/auxiliares de enfermagem).

Ressalta-se que, apesar do estudo realizado demonstrar que, pela aplicação do modelo de Gaidzinski ${ }^{(1)}$, o quadro de pessoal de enfermagem do HSP, com alguns ajustes, é compatível com a necessidade de assistência aos pacientes internados, permanece ainda a percepção das enfermeiras quanto à insuficiência de pessoal no quadro existente. As causas atribuídas a esta percepção são: inconstância do número e grau de complexidade dos pacientes internados na maioria das unidades; falta de alocação de pessoal para cobrir licenças médicas prolongadas e licenças maternidade; e morosidade na reposição das vagas decorrentes de desligamentos e aposentadorias.

Um estudo semelhante realizado no Hospital Universitário da Universidade de São Paulo, sugere que, para corrigir a distorção provocada pela distribuição irregular do número de pacientes internados, podem ser incorporados ao cálculo, além da média diária de pacientes, o desvio padrão e a mediana das amostras diárias do número de pacientes ${ }^{(6)}$. A soma do desvio padrão, segundo a autora, adequa o resultado, melhorando o provimento de pessoal para a assistência de enfermagem, porém este recurso só deve ser empregado em unidades onde a ocupação dos leitos não é de $100 \%$.

\section{CONCLUSÕES}

Face aos resultados encontrados neste estudo, concluiu-se que:

- Quanto aos níveis de complexidade dos cuidados de enfermagem, apesar do Hospital São Paulo ser categorizado como de nível terciá-rio, os pacientes internados demandaram principalmente de cuidados mínimos $(30,8 \%)$; seguidos pelos intermediários $(27,5 \%)$; cuidados de alta dependência $(22,0 \%)$; cuidados intensivos $(12,0 \%)$ e cuidados semi-intensivos (7,6\%). A distribuição dos pacientes segundo este sistema de classificação foi diversificada nas unidades de internação, com algumas exceções, dificultando o provimento adequado 
de pessoal de enfermagem na rotina diária destas unidades;

- o tempo destinado à prestação da assistência de enfermagem aos pacientes classificados foi maior para os pacientes que necessitavam de cuidados intensivos (30,9\%), seguidos pelos de alta dependência $(21,8 \%)$, intermediários $(21,2 \%)$, mínimos $(15,3 \%)$ e semi-intensivos $(10,7 \%)$;

- cerca de 50,0\% das ausências não previstas das enfermeiras foram referentes às licenças maternidade. A distribuição destas ausências para os técnicos/auxiliares de enfermagem foi homogênea entre faltas, licenças médicas, licenças maternidade e outras;

- o dimensionamento do pessoal de enfermagem realizado segundo o modelo de Gaidzinski ${ }^{(1)}$ indicou um total de 337 enfermeiras e 557 técnicos/auxiliares de enfermagem. A comparação entre os quadros de pessoal de enfermagem existente no HSP e o projetado, demonstrou carência de 76 enfermeiras e excesso de 97 técnicos/auxiliares de enfermagem.

A análise das variáveis intervenientes no processo de dimensionamento do pessoal de enfermagem do HSP apontou a necessidade de reorganização do processo de trabalho referente ao agrupamento de pacientes por complexidade assistencial; e a diminuição do valor do acréscimo relativo às ausências.

Ressalta-se a importância da realização de novos estudos, a fim de obter subsídios para diminuir as ausências programadas e não programadas, assim como comparar os resultados obtidos com hospitais de porte e complexidade semelhantes

\section{REFERÊNCIAS}

1. Gaidzinski RR. O dimensionamento de pessoal de enfermagem em instituições hospitalares. [tese doutorado] São Paulo :Escola de Enfermagem da USP; 1998.

2. Quintana PB, Roscheke MAC, Ribeiro ECO. Educaciòn permanente, processo de trabalho y calidad de servicio en salud. In: Haddad QJ, Roscheke MAC, Davini MC, editores. Educaciòn permanente de personal de salud. Washington: OPS; 1994. p.33-61.

3. Magalhães AMM, Duarte ERM, Moura GMSS. Estudo das variáveis que participam do dimensionamento de pessoal de enfermagem em hospitais de grande porte. Rev Gaúcha Enferm 1995;16 (1/2):5-16.

4. Kurcgant P, Cunha K, Gaidzinski RR. Subsídios para a estimativa de pessoal de enfermagem. Enfoque 1989; 17(3): 79-81.

5. Gaidzinski RR. O dimensionamento do pessoal de enfermagem segundo a percepção de enfermeiras que vivenciam esta prática. [tese doutorado] São Paulo: Escola de Enfermagem da USP; 1994.

6. Fugulin FMT. Dimensionamento de pessoal de enfermagem: avaliação do quadro de pessoal das unidades de internação de um hospital de ensino. [tese doutorado] São Paulo: Escola de Enfermagem da USP; 2002.

7. Alcalá UM, Nunes MF, KatoT, Reigada I, Silva RML, Yoshimura DK. Cálculo de pessoal: estudo peliminar para o estabelecimento de quadro de pessoal de enfermagem na superintendência médico hospitalar de urgência. São Paulo: Secretaria de Higiene e Saúde; 1982.

8. Fugulin FMT, Silva SHS, Shimizu HE, Campos FPF. Implantação do sistema de classificação de pacientes na unidade de clínica médica do hospital universitário da USP. Rev Med HU-USP 1994; 4(1/2): 63-8.
9. Conselho Federal de Enfermagem. Resolução no 189/96. Estabelece parâmetros para dimensionamento do quadro de profissionais de enfermagem nas instituições de saúde.In: Conselho Regional de Enfermagem. Documentos básicos de enfermagem: enfermeiros, técnicos e auxiliares. São Paulo; 2001. p.144-51.

10. Campedelli MC, Takito C, Sancinetti TR, Benko MA. Cálculo de pessoal de enfermagem: competência da enfermeira. Rev Esc Enferm USP 1987;21(1):3-15.

11. Gaidzinski RR. Dimensionamento de pessoal de enfermagem. In:Kurcgant P, coordenadora. Administração em enfermagem. São Paulo: EPU; 1991. cap.7, p.91-6.

12. Ide P, Kirby KK, Stark MS. Operation room productivity. An evaluation format. J Nurs Adm 1992; 22(10): 41-8.

13. Associação Americana de Hospitais/ Liga Nacional de Educação em Enfermagem. Manual de fundamentos da boa enfermagem hospitalar. Trad. de Serviço Especial de Saúde Pública. Rio de Janeiro; 1945. Qualidade e quantidade do serviço de enfermagem; cap.11,p.40-50.

14. Perroca MG. Sistema de classificação de pacientes: construção e validação de um instrumento.[dissertação mestrado] São Paulo: Escola de Enfermagem da USP; 1996.

15. Jorge AL. Motivos que levam os trabalhadores de enfermagem ao absenteísmo. Acta Paul Enferm 1995; 8(1):39-46. 
Matsushita MS, Adami NP, Carmagnani MIS. [Nursing staff dimensioning in the hospitalization units of the Hospital São Paulo.] Acta Paul Enferm 2005; 18(1):9-19.

ABSTRACT:This descriptive study was carried out at the hospital São Paulo aiming nursing staff dimension of the hospitalization units of this Institution. Data collect was performed in 2002, in the 40 hospitalization units in which patient's classification and non anticipated absences of the nursing staff of the previous year were studied, applying the staff dimensioning calculation proposed by Gaidzinski(1). The main conclusions were regarding levels of complexity of the nursing care, the hospitalized patients demanded minimum care of nursing $(30.8 \%)$ followed by intermediary care $(27.5 \%)$, high dependence care $(22.0 \%)$, intensive care $(12 \%)$ and semi-intensive care $(7.6 \%)$. Time of nursing assistance for the classified patients was greater for intensive care $(30.9 \%)$ followed by high dependence $(21.8 \%)$, intermediary $(21.2 \%)$ minimum $(15.3 \%)$ and semi-intensive $(10.7 \%)$. Almost $50.0 \%$ of the non-anticipated absences were due to maternity permissions and those of nursing technicians/assistants and others were homogeneous as no show at work, medical authorization, maternity permission and others. The comparison between the nursing staff in the Hospital São Paulo and the calculated according to the Gaidzinski model showed a need of 76 nurses and superlative of 97 nursing technician/assistants.

Descriptors: Nursing staff; Nursing staff, hospital; Personnel turnover; Personnel administration; hospital. Personnel downsizing
Matsushita MS, Adami NP, Carmagnani MIS. [Dimensionamiento del personal de enfermería de las unidades de internación del Hospital São Paulo.] Acta Paul Enferm 2005; 18(1):9-19.

RESUMEN: Este estudio descriptivo fue realizado en el Hospital São Paulo con el objetivo de dimensionar el personal de enfermería de las unidades de internación de esta institución. La colecta de datos fue realizada en el año 2002, en las 40 unidades de internación, donde se estudió la clasificación de pacientes y las ausencias no previstas de los profesionales de enfermería de año anterior, siendo aplicado el cálculo de dimensionamiento del personal propuesto por Gaidzinski (1). Las principales conclusiones fueron: cuanto a los niveles de complejidad de los cuidados de enfermería, los pacientes internados demandaran de cuidados mínimos $(30.8 \%)$, seguidos por los cuidados intermediarios $(27.5 \%)$, cuidados de alta dependencia $(22.0 \%)$, cuidados intensivos (12\%) y cuidados seme-intensivos (7.6\%). El tiempo de asistencia de enfermería destinado a los pacientes clasificados fue mayor para los cuidados intensivos $(30.9 \%)$, seguidos por los de alta dependencia (21.8\%), intermediarios $(21.2 \%)$, mínimos (15.3\%) y seme-intensivos $(10.7 \%)$. Cerca de $50.0 \%$ de las ausencias no previstas de las enfermeras fueron referentes a las licencias de maternidad a las de los técnicos / auxiliares de enfermería fueron homogéneas entre faltas, licencias médicas, licencias de maternidad y otras. La comparación entre los cuadros de personal de enfermería existente en el Hospital São Paulo y lo calculado según el modelo de Gaidzinski demostró carencia de 76 enfermeras y superávit de 97 técnicos / auxiliares de enfermería.

Descriptores: Personal de Enfermería; Personal de enfermería en hospital; Reorganización del personal; Administración de personal en hospitales; Reducción de personal 